\title{
Nucleon sigma terms for $2+1$ quark flavours
}

\section{R. Horsley ${ }^{* a}$, Y. Nakamura ${ }^{b}$, H. Perlt ${ }^{c}$, D. Pleiter ${ }^{d e}$, P. E. L. Rakow ${ }^{f}$, G. Schierholz ${ }^{e h}$,} A. Schiller ${ }^{c}$, H. Stüben ${ }^{i}$, F. Winter ${ }^{a}$ and J. M. Zanotti ${ }^{a}$

${ }^{a}$ School of Physics and Astronomy, University of Edinburgh, Edinburgh EH9 3JZ, UK

${ }^{b}$ RIKEN Advanced Institute for Computational Science, Kobe, Hyogo 650-0047, Japan

${ }^{c}$ Institut für Theoretische Physik, Universität Leipzig, 04109 Leipzig, Germany

${ }^{d}$ Jülich Research Centre, JSC, Room 324, 52425 Jülich, Germany

${ }^{e}$ Institut für Theoretische Physik, Universität Regensburg, 93040 Regensburg, Germany

${ }^{f}$ Theoretical Physics Division, Department of Mathematical Sciences, University of Liverpool, Liverpool L69 3BX, UK

${ }^{h}$ Deutsches Elektronen-Synchrotron DESY, 22603 Hamburg, Germany

${ }^{i}$ Konrad-Zuse-Zentrum für Informationstechnik Berlin, 14195 Berlin, Germany

E-mail: rhorsleyeph.ed.ac.uk

\section{QCDSF-UKQCD Collaboration}

QCD lattice simulations yield hadron masses as functions of the quark masses. From the gradients of the hadron masses the sigma terms can then be determined. We consider here dynamical $2+1$ flavour simulations, in which we start from a point of the flavour symmetric line and then keep the singlet or average quark mass fixed as we approach the physical point. This leads to highly constrained fits for hadron masses in a multiplet. The gradient of this path for a hadron mass then gives a relation between the light and strange sigma terms. A further relation can be found from the change in the singlet quark mass along the flavour symmetric line. This enables light and strange sigma terms to be estimated for the baryon octet.

The XXIX International Symposium on Lattice Field Theory - Lattice 2011

July 10-16, 2011

Squaw Valley, Lake Tahoe, California

\footnotetext{
* Speaker.
} 


\section{Introduction}

In this talk we shall describe a method for the determination of the hyperon sigma terms based on the results of [1] to which we refer to for more details including numerical results.

Sigma terms, $\sigma_{l}^{(H)}, \sigma_{s}^{(H)}$ are defined as that part of the mass of the hadron (for example the nucleon) coming from the vacuum connected expectation value of the up $(u)$ down $(d)$ and strange $(s)$ quark mass terms in the QCD Hamiltonian,

$$
\sigma_{l}^{(H)}=m_{l}^{R}\left\langle H\left|(\bar{u} u+\bar{d} d)^{R}\right| H\right\rangle, \quad \sigma_{s}^{(H)}=m_{s}^{R}\left\langle H\left|(\bar{s} s)^{R}\right| H\right\rangle,
$$

where we have taken the $u$ and $d$ quarks to be mass degenerate, $m_{u}=m_{d} \equiv m_{l}$. (The superscript ${ }^{R}$ denotes a renormalised quantity.) Eq. (1.1) is usually written (in particular for the nucleon) as

$$
\sigma_{l}^{(N)}=\frac{m_{l}^{R}\left\langle N\left|(\bar{u} u+\bar{d} d-2 \bar{s} s)^{R}\right| N\right\rangle}{1-y^{(N) R}}, \quad y^{(N) R}=\frac{2\left\langle N\left|(\bar{s} s)^{R}\right| N\right\rangle}{\left\langle N\left|(\bar{u} u+\bar{d} d)^{R}\right| N\right\rangle},
$$

(i.e. we consider $y^{(N)_{R}}$ rather than $\sigma_{s}^{(N)}$ ). The simplest calculation, (which we will discuss in more detail later) uses first order in $S U(3)$ flavour symmetry (octet) breaking to give

$$
\sigma_{l}^{(N)}=\frac{m_{l}^{R}}{m_{s}^{R}-m_{l}^{R}} \frac{M_{\Xi}+M_{\Sigma}-2 M_{N}}{1-y^{(N) R}} \sim \frac{26}{1-y^{(N) R}} \mathrm{MeV},
$$

and

$$
\sigma_{s}^{(N)}=\frac{m_{s}^{R}}{m_{l}^{R}} \frac{1}{2} y^{(N) R} \sigma_{l}^{(N)} \sim 325 \frac{y^{(N) R}}{1-y^{(N) R}} \mathrm{MeV},
$$

where $m_{s}^{R} / m_{l}^{R}$ is the ratio of the strange to light quark masses, which using the leading order PCAC formula for this ratio gives $m_{s}^{R} / m_{l}^{R}=\left(2 M_{K}^{2}-M_{\pi}^{2}\right) / M_{\pi}^{2} \sim 25$. The Zweig rule, $\left\langle N\left|(\bar{s} s)^{R}\right| N\right\rangle \sim 0$ would then give $\sigma_{l}^{(N)} \sim 26 \mathrm{MeV}, \sigma_{s}^{(N)} \sim 0 \mathrm{MeV}$ while any non-zero strangeness content, $y^{(N) R}>0$ would increase this value of $\sigma_{l}^{(N)}, \sigma_{s}^{(N)}$ (and indeed due to the large coefficient, $\sigma_{s}^{(N)}$ quite rapidly).

Computing the sigma terms from lattice QCD has a long history from quenched to 2 flavour and more recently $2+1$ flavour simulations. In general more recent results tend to give lower values than earlier determinations.

In this talk, we shall investigate this simple picture as described above and in particular test the linearity assumption of $S U(3)$ flavour symmetry breaking.

\section{Flavour symmetry expansions}

Lattice simulations start at some point in the $\left(m_{s}^{R}, m_{l}^{R}\right)$ plane and then approach the physical point $\left(m_{s}^{R *}, m_{l}^{R *}\right)$ along some path. (In future we shall denote the physical point with a ${ }^{*}$.) As we shall be considering flavour symmetry breaking then we shall start here at a point on the flavour symmetric line $m_{l}^{R}=m_{s}^{R}$ and then consider the path keeping the average quark mass constant, $\bar{m}=$ const.. The $S U$ (3) flavour group (and quark permutation symmetry) then restricts the quark mass polynomials that are allowed, giving for the baryon octet

$$
M_{H}=M_{0}(\bar{m})+c_{H} \delta m_{l}+O\left(\delta m_{l}^{2}\right),
$$


with $c_{N}=3 A_{1}, c_{\Lambda}=3 A_{2}, c_{\Sigma}=-3 A_{2}, c_{\Xi}=-3\left(A_{1}-A_{2}\right)$ and

$$
\delta m_{l}=m_{l}-\bar{m}, \quad \bar{m}=\frac{1}{3}\left(2 m_{l}+m_{s}\right) .
$$

So to linear order in the quark mass, we only have two unknowns, $A_{1}, A_{2}$ (rather than four). A similar situation also holds for the pseudoscalar and vector octets (one unknown) and baryon decuplet (also one unknown). This highly constrains the numerical fits.

Permutation invariant functions of the masses, $X_{S}$, (or 'centre of mass' of the multiplet) have no linear dependence on the quark mass. For example for the baryon octet we have

$$
X_{N}=\frac{1}{3}\left(M_{N}+M_{\Sigma}+M_{\Xi}\right)=M_{0}(\bar{m})+O\left(\delta m_{l}^{2}\right) .
$$

(The corresponding result for the pseudoscalar octet is given later in eq. (3.10).)

Furthermore expanding about a specific fixed point, $m_{l}=m_{s}=m_{0}$ on the flavour symmetric line and allowing $\bar{m}$ to vary, we then have

$$
M_{0}(\bar{m})=M_{0}\left(m_{0}\right)+M_{0}^{\prime}\left(m_{0}\right)\left(\bar{m}-m_{0}\right)+O\left(\left(\bar{m}-m_{0}\right)^{2}\right) .
$$

We will see that $A_{1}, A_{2}$ determine all the non-singlet sigma terms and $M^{\prime}\left(m_{0}\right)$ the singlet sigma terms.

As an example of the quark mass expansion from a point on the flavour symmetric line in Fig. 1 we plot the baryon octet $M_{H} / X_{N}$ for $H=N, \Lambda, \Sigma, \Xi$ against $M_{\pi}^{2} / X_{\pi}^{2}$ together with a linear fit,
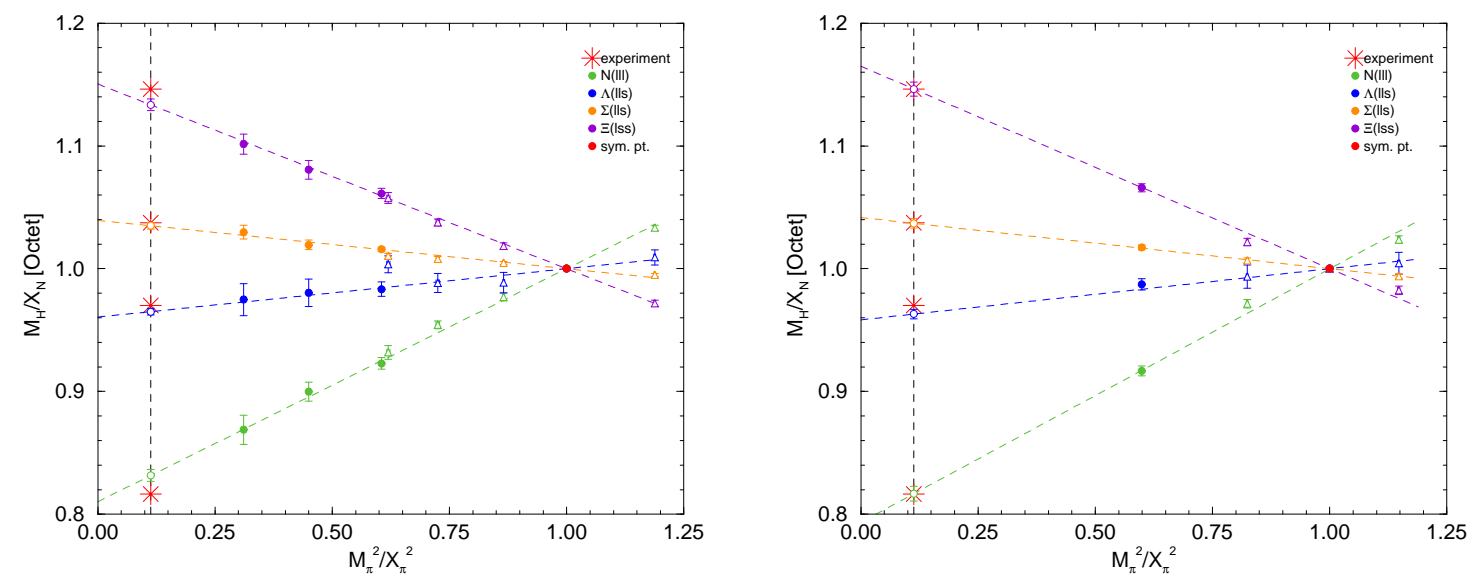

Figure 1: $M_{H} / X_{N}(H=N, \Lambda, \Sigma, \Xi)$ against $M_{\pi}^{2} / X_{\pi}^{2}$ for initial point on the flavour symmetric line given by $\kappa_{0}=0.12090$, left panel and $\kappa_{0}=0.12092$, right panel. The $32^{3} \times 64$ lattices are filled circles, while the $24^{3} \times 48$ lattices are open triangles. Also shown is the combined fit of eq. (3.11) (the dashed lines) to the $32^{3} \times 64$ lattice data. The fit results are the open circles, while the experimental points are the (red) stars.

eq. (2.1) and implicitly eq. (3.10) using $2+1 O(a)$ improved clover fermions at $\beta=5.50$, using two starting values for the quark mass on the flavour symmetric line. All the points have been arranged in the simulation to have constant $\bar{m}$. We see that a linear fit provides a good description of the numerical data from the symmetric point (where $M_{\pi} \sim X_{\pi}^{*}=410.9 \mathrm{MeV}$ ) down to the physical pion mass. 


\section{3. (Hyperon) $\sigma$ equations}

\subsection{Renormalisation}

For Wilson (clover) fermions under renormalisation the singlet and non-singlet pieces of the quark mass renormalise differently. We have

$$
m_{q}^{R}=Z^{N S}\left[m_{q}+\alpha_{Z} \frac{1}{3}\left(2 m_{l}+m_{s}\right)\right], \quad \alpha_{Z}=\frac{Z^{N S}-Z^{S}}{Z^{N S}} .
$$

In the action the term $\sum_{q} m_{q} \bar{q} q=\sum_{q} m_{q}^{R}(\bar{q} q)^{R}$ i.e. is a renormalisation group invariant or RGI quantity. Upon writing this in a matrix form and inverting gives

$$
(\bar{q} q)^{R}=\frac{1}{Z^{N S}}\left[\bar{q} q-\frac{\alpha_{Z}}{1+\alpha_{Z}} \frac{1}{3}(\bar{u} u+\bar{d} d+\bar{s} s)\right],
$$

so for $\alpha_{Z} \neq 0$ then there is always mixing between bare operators. Useful quark combinations are the octet ${ }^{1}$ and singlet combinations, namely

$$
\begin{aligned}
(\bar{u} u+\bar{d} d)^{R}-2(\bar{s} s)^{R} & =\frac{1}{Z^{N S}}((\bar{u} u+\bar{d} d)-2(\bar{s} s)) \\
(\bar{u} u+\bar{d} d)^{R}+(\bar{s} s)^{R} & =\frac{1}{Z^{N S}\left(1+\alpha_{Z}\right)}((\bar{u} u+\bar{d} d)+(\bar{s} s)) .
\end{aligned}
$$

\section{$3.2 \sigma$ equations}

Scalar matrix elements can be determined from the gradient of the hadron mass (with respect to the quark mass) by using the Feynman-Hellman theorem which is true for both bare and renormalised quantities. So if we take the derivative with respect to the bare quark mass we get the bare $\bar{q} q$ matrix element,

$$
\frac{\partial M_{H}}{\partial m_{l}}=\langle H|(\bar{u} u+\bar{d} d)| H\rangle, \quad \frac{\partial M_{H}}{\partial m_{s}}=\langle H|\bar{s} s| H\rangle .
$$

Multiplying the renormalised quark mass, eq. (3.1), together with eqs. (3.4) (or more generally with eq. (3.2)) we can find RGI combinations (i.e. a form where the renormalisation constant $Z^{N S}$ cancels). In particular we find

$$
\begin{aligned}
\sigma_{l}^{(H)}-2 r \sigma_{s}^{(H)} & =\frac{3 r}{1+2 r}\left(1+\alpha_{Z}\right) m_{0} c_{H} \\
\sigma_{l}^{(H)}+r \sigma_{s}^{(H)} & =\frac{3 r}{1+2 r} m_{0} M_{0}^{\prime}\left(m_{0}\right),
\end{aligned}
$$

where $r$ is the ratio of quark masses $r \equiv m_{l}^{R} / m_{s}^{R}$. The two simultaneous equations, which can be easily solved, give

$$
\begin{aligned}
\sigma_{l}^{(H)} & =\frac{r}{1+2 r}\left[\left(1+\alpha_{Z}\right) m_{0} c_{H}+2 m_{0} M_{0}^{\prime}\left(m_{0}\right)\right] \\
\sigma_{s}^{(H)} & =\frac{1}{1+2 r}\left[-\left(1+\alpha_{Z}\right) m_{0} c_{H}+m_{0} M_{0}^{\prime}\left(m_{0}\right)\right] .
\end{aligned}
$$

\footnotetext{
${ }^{1}$ Eq. (3.3) also leads to eq. (1.3). The RHS of eq. (3.3) can be re-written as $c_{N} / Z^{N S}=3 A_{1} / Z^{N S}$. Together with $M_{\Xi}+M_{\Sigma}-2 M_{N}=-9 A_{1} \delta m_{l}=3 A_{1}\left(m_{s}^{R}-m_{l}^{R}\right) / Z^{N S}$ this gives eq. 1.3). An alternative mass combination that also picks out the $A_{1}$ coefficient is $M_{\Xi}-M_{\Lambda}=-3 A_{1} \delta m_{l}$.
} 
We see that the smallness of $\sigma_{l}^{(H)}$ in comparison to $\sigma_{s}^{(H)}$ is certainly guaranteed by the presence of an additional $r$ in its numerator. These coefficients are also sufficient to determine $y^{(H) R}$, as can be seen from eq. (3.8),

$$
y^{(H) R}=2 \frac{-\left(1+\alpha_{Z}\right) m_{0} c_{H}+m_{0} M_{0}^{\prime}\left(m_{0}\right)}{\left(1+\alpha_{Z}\right) m_{0} c_{H}+2 m_{0} M_{0}^{\prime}\left(m_{0}\right)} .
$$

It is seen that $y^{(H) R}$ only depends on gradients and not on the physical point.

It is convenient to normalise the coefficients by $X_{N}$ so we now need to find the coefficients $\left(1+\alpha_{Z}\right) m_{0} c_{H} / X_{N}\left(m_{0}\right)$ and $m_{0} M_{0}^{\prime}\left(m_{0}\right) / X_{N}\left(m_{0}\right)$ and also to extrapolate to the point where the quark mass ratio takes its physical value, i.e. $r=r^{*}$.

\subsection{Determination of the coefficients}

The coefficients can be determined by considering gradients with respect to a physical quantity. As in eq. (2.1) we also have a similar expansion for the pseudoscalar octet,

$$
M_{\pi}^{2}=M_{0 \pi}^{2}+2 \alpha \delta m_{l}+O\left(\delta m_{l}^{2}\right),
$$

(together with $M_{K}^{2}=M_{0 \pi}^{2}-\alpha \delta m_{l}+O\left(\delta m_{l}^{2}\right), M_{\eta_{s}}^{2}=M_{0 \pi}^{2}-4 \alpha \delta m_{l}+O\left(\delta m_{l}^{2}\right)$ ). Analogously to eq. (2.3) we can define a flavour singlet quantity $X_{\pi}^{2}=\frac{1}{3}\left(2 M_{K}^{2}+M_{\pi}^{2}\right)=M_{0 \pi}^{2}+O\left(\delta m_{l}^{2}\right)$ However, as well as eq. (2.1), we have the additional constraint from PCAC $M_{\pi}^{2}=2 B_{0}^{R} m_{l}^{R}$ (together with $\left.M_{K}^{2}=B_{0}^{R}\left(m_{l}^{R}+m_{s}^{R}\right)\right)$. If we now consider an expansion in the pion mass then eliminating $\delta m_{l}$ between eq. (2.1) and eq. (3.10) gives

$$
\frac{M_{H}}{X_{N}}=\left(1-\left[\left(1+\alpha_{Z}\right) m_{0} \frac{c_{H}}{X_{N}}\right]\right)+\left[\left(1+\alpha_{Z}\right) m_{0} \frac{c_{H}}{X_{N}}\right] \frac{M_{\pi}^{2}}{X_{\pi}^{2}},
$$

from the point on the symmetric line $m_{0}=\bar{m}$. Thus if we plot $M_{H} / X_{N}$ versus $M_{\pi}^{2} / X_{\pi}^{2}$ (holding the singlet quark mass, $\bar{m}$ constant) then the gradient immediately yields $\left(1+\alpha_{Z}\right) m_{0} c_{H} / X_{N}$. The only assumption is that the 'fan' plot splittings remain linear in $\delta m_{l}$ down to the physical point. In Fig. 11 we show this plot leading to a results for $\left(1+\alpha_{Z}\right) m_{0} c_{H} / X_{N}$ for $\kappa_{0}=0.12090,0.12092$.

Furthermore on the flavour symmetric line eliminating $\left(\bar{m}-m_{0}\right)$ between eqs. (2.4) and the corresponding one for $M_{\pi}^{2}(\bar{m})$ gives

$$
\frac{X_{N}(\bar{m})}{X_{N}\left(m_{0}\right)}=\left(1-\left[\frac{m_{0} M_{0}^{\prime}\left(m_{0}\right)}{X_{N}\left(m_{0}\right)}\right]\right)+\left[\frac{m_{0} M_{0}^{\prime}\left(m_{0}\right)}{X_{N}\left(m_{0}\right)}\right] \frac{X_{\pi}^{2}(\bar{m})}{X_{\pi}^{2}\left(m_{0}\right)} .
$$

Again in a plot of $X_{N}(\bar{m}) / X_{N}\left(m_{0}\right)$ versus $X_{\pi}^{2}(\bar{m}) / X_{\pi}^{2}\left(m_{0}\right)$ the gradient immediately gives the required ratio $m_{0} M_{0}^{\prime}\left(m_{0}\right) / X_{N}\left(m_{0}\right)$. In Fig. 目 we plot $X_{N}(\bar{m}) / X_{N}\left(m_{0}\right)$ versus $X_{\pi}^{2}(\bar{m}) / X_{\pi}^{2}\left(m_{0}\right)$. From eq. (3.12) the gradient gives the required number.

Finally the quark mass ratio, $r$, must be estimated. In the right panel of Fig. 2 we plot $\left(2 M_{K}^{2}-\right.$ $\left.M_{\pi}^{2}\right) / X_{N}^{2}$ versus $M_{\pi}^{2} / X_{N}^{2}$. From eq. (3.10) we have

$$
\frac{2 M_{K}^{2}-M_{\pi}^{2}}{X_{N}^{2}}=3 \frac{M_{0 \pi}^{2}}{X_{N}^{2}}-2 \frac{M_{\pi}^{2}}{X_{N}^{2}} .
$$

As in section 2, we see that for constant $\bar{m}$ the data points lie on a straight line (i.e. there is an absence of significant non-linearity). Together with PCAC, this gives the $x$-axis is proportional to 

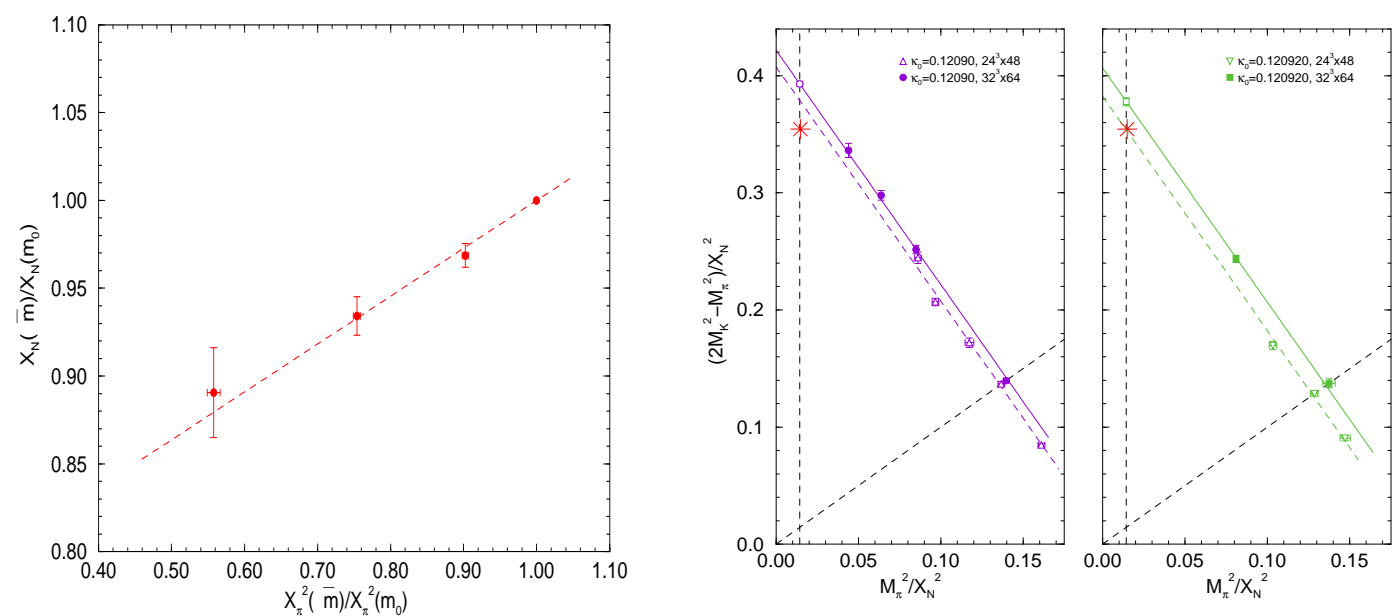

Figure 2: Left plot: $X_{N}(\bar{m}) / X_{N}\left(m_{0}\right)$ versus $X_{\pi}^{2}(\bar{m}) / X_{\pi}^{2}\left(m_{0}\right)$ along the flavour symmetric line, together with the linear fit from eq. (3.12); Right plot: $\left(2 M_{K}^{2}-M_{\pi}^{2}\right) / X_{N}^{2}$ versus $M_{\pi}^{2} / X_{N}^{2}$ for $\kappa_{0}=0.12090$ (left panel) and $\kappa_{0}=0.12092$ (right panel). The $32^{3} \times 64$ volume results are given by the filled symbols, while the $24^{3} \times 48$ volume results are shown using empty triangles. The fit is given in eq. (3.13). Experimental points are denoted by (red) stars.

$m_{l}^{R}$ while the $y$-axis is proportional to $m_{s}^{R}$ and thus the ratio gives $r$. Taking our physical scale to be defined from $M_{\pi}^{2} /\left.X_{N}^{2}\right|^{*}$ (i.e. from the $x$-axes of Fig. 国) gives $1 / r^{*}$.

What can we say about corrections to the linear terms? The simple linear fit describes the data well, from the symmetric point to our lightest pion mass, both along the $\bar{m}=$ const. line and the flavour symmetric line. To see the possible influence of curvature we compare linear fits with quadratic fits as discussed in the Appendix of [1]. These will be used in section 4 for the estimate of possible systematic effects.

\section{Results and Conclusions}

We can now determine $y^{(H) R}$ and $\sigma_{l}^{(H)}, \sigma_{s}^{(H)}$. The scale is taken as $X_{N}=1.1501 \mathrm{GeV}$. We shall only discuss here the general details of the results; the numerical values are given in [1].

From eq. (3.6) we can find an indication of the magnitude of $\sigma_{l}^{(N)}$ as approximately

$$
\sigma_{l}^{(N) *} \sim 22.4+\frac{\sigma_{s}^{(N) *}}{13.6} \mathrm{MeV} \gtrsim 22.4 \mathrm{MeV}
$$

The last inequality follows as obviously $\sigma_{s}^{(N) *}>0$. Indeed this shows that a non-zero $\sigma_{s}^{(N) *}>0$ can only add a few $\mathrm{MeV}$ to this result.

These results are illustrated in the left plot of Fig. 目 for $y^{(H) R *}$ and in the right plots $\sigma_{l}^{(H) *}$, $\sigma_{s}^{(H) *}$ both against $H=N, \Lambda, \Sigma$ and $\Xi$. While the data for $\kappa_{0}=0.12090$ is more complete than for $\kappa_{0}=0.12092$ (cf. the plots in Fig. 1) and demonstrates linear behaviour, as the path starting at $\kappa_{0}=0.12092$ is closer to the physical point (cf. Fig. 2) we shall use these values as our final values.

In conclusion we have found that keeping the average quark mass constant gives very linear 'fan' plots from the flavour symmetric point down to the physical point. This implies that an 

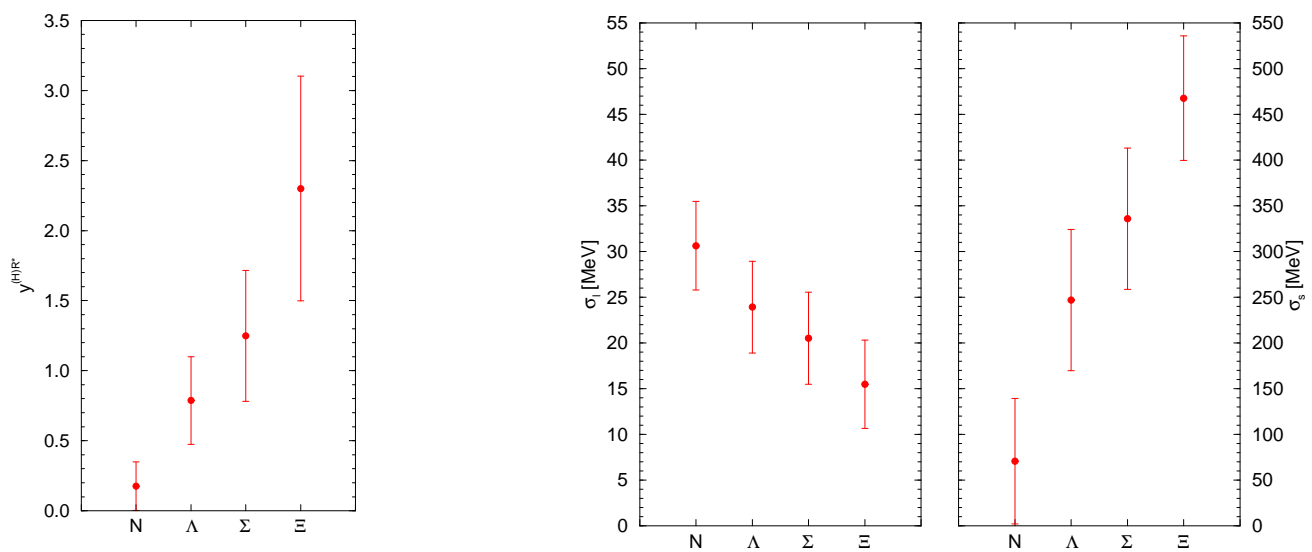

Figure 3: $y^{(H) R *}$ (left plot) and $\sigma_{l}^{(H) *}$ and $\sigma_{s}^{(H) *}$ (right plots) both at the physical point for $H=N, \Lambda, \Sigma, \Xi$

expansion in the quark mass from the flavour symmetric point will give information about the physical point. In this talk we have applied this to estimating the sigma terms (both light and strange) of the nucleon octet. There has been no use of a chiral perturbation expansion (indeed this is an opposite expansion to the one used here, expanding about zero quark mass).

Note that expansions about the $S U(3)$ flavour line require consistency between many QCD observables, here for example not only for the baryon octet under consideration here, but also for the pseudoscalar octet, and PCAC and the ratio of the light to strange quark mass.

Of course there are several more avenues to investigate. Our approach here has been to emphasise linearity at the expense (presently) of reaching exactly the physical point. This can be addressed by interpolating between a small set of constant $\bar{m}$ lines about the physical point. Additionally the use of partial quenching will also help to get closer to the physical pion mass. With more data, a systematic investigation of quadratic quark mass terms in the flavour expansion should be considered, to reduce the systematic errors.

\section{Acknowledgements}

The numerical configuration generation was performed using the BQCD lattice QCD program, on the IBM BlueGeneL at EPCC (Edinburgh, UK), the BlueGeneL and P at NIC (Jülich, Germany), the SGI ICE 8200 at HLRN (Berlin-Hannover, Germany) and the JSCC (Moscow, Russia). We thank all institutions. The BlueGene codes were optimised using Bagel. The Chroma software library, was used in the data analysis. This work has been supported in part by the EU grants 227431 (Hadron Physics2), 238353 (ITN STRONGnet) and by the DFG under contract SFB/TR 55 (Hadron Physics from Lattice QCD). JMZ is supported by STFC grant ST/F009658/1.

\section{References}

[1] R. Horsley et al., [QCDSF-UKQCD Collaboration], arXiv:1110.4971. 